TITLE:

\title{
Brief report: Representational momentum for dynamic facial expressions in pervasive developmental disorder.
}

\section{$\operatorname{AUTHOR}(\mathrm{S})$ :}

Uono, Shota; Sato, Wataru; Toichi, Motomi

\section{CITATION:}

Uono, Shota ... [et al]. Brief report: Representational momentum for dynamic facial expressions in pervasive developmental disorder.. Journal of autism and developmental disorders 2010, 40(3): 371-377

\section{ISSUE DATE:}

2010-03

URL:

http://hdl.handle.net/2433/128900

\section{RIGHT:}

The original publication is available at www.springerlink.com; この論文 は出版社版でありません。引用の際には出版社版をご確認ご利用くだ さい。; This is not the published version. Please cite only the published version. 
doi: 10.1007/s10803-009-0870-9

\section{Brief Report: Representational Momentum for Dynamic Facial}

\section{Expressions in Pervasive Developmental Disorder}

Shota Uono ${ }^{1}$, Wataru Sato $^{2}$, and Motomi Toichi ${ }^{3}$

${ }^{1}$ Department of Cognitive Psychology in Education, Faculty of Education, Kyoto University, Kyoto, Japan

${ }^{2}$ Department of Comparative Study of Cognitive Development (Funded by Benesse Corporation), Primate Research Institute, Kyoto University, Inuyama, Japan

${ }^{3}$ Faculty of Human Health Science, Graduate School of Medicine, Kyoto University, Kyoto, Japan

Running Head: Representational Momentum in PDD 


\begin{abstract}
Individuals with pervasive developmental disorder (PDD) have difficulty with social communication via emotional facial expressions, but behavioral studies involving static images have reported inconsistent findings about emotion recognition. We investigated whether dynamic presentation of facial expression would enhance subjective perception of expressed emotion in 13 individuals with PDD and 13 typically developing controls. We presented dynamic and static emotional (fearful and happy) expressions. Participants were asked to match a changeable emotional face display with the last presented image. The results showed that both groups perceived the last image of dynamic facial expression to be more emotionally exaggerated than the static facial expression. This finding suggests that individuals with PDD have an intact perceptual mechanism for processing dynamic information in another individual's face.
\end{abstract}

Keywords: Asperger's disorder; Dynamic facial expression; Pervasive developmental disorder; Representational momentum

Correspondence: shota-uono@p06.mbox.media.kyoto-u.ac.jp 


\section{Introduction}

Individuals with pervasive developmental disorder (PDD) have difficulty with social interaction, including communication via emotional facial expressions (American Psychiatric Association [APA] 2000). Over the last two decades, many experimental studies have investigated this issue using static faces as stimuli, but results have been contradictory. For example, some studies showed that individuals with PDD had a normal ability to discriminate (e.g., Adolphs et al. 2001) and recognize (e.g., Castelli 2005) the facial expressions of others. Other studies reported that these individuals were impaired in the recognition of facial expressions, particularly facial expressions of a fearful emotional state (e.g., Corden et al. 2008).

Social interactions in daily life are mainly based on dynamic facial cues. Consistent with this notion, some studies in typically developing subjects have reported that dynamic facial expressions enhance various types of processing, including expression perception (Yoshikawa and Sato 2008), emotion recognition (Bould et al. 2008) and emotional reactions (Sato and Yoshikawa 2007), compared to static facial expressions. Use of dynamic, relative to static, face stimuli may provide ecologically valid evidence on the facial expression processing in individuals with PDD.

A few previous studies have explored the recognition of dynamic facial expressions in individuals with PDD. Gepner et al. (2001) investigated this issue using dynamic, strobe flash, and static presentations. Although individuals with PDD were as able as typically developing controls to recognize dynamic and static facial expressions, strobe flash presentation improved recognition of facial expression compared with static presentation in typically developing controls but not in individuals with PDD. Tardif et al. (2007) demonstrated that slow dynamic presentation of facial expressions improved emotion recognition in individuals with PDD, although these participants were less able than were controls to recognize dynamic and static facial expressions. 
Previous studies therefore suggest differences in performance between individuals with PDD and normal controls in the recognition of dynamic facial expression. However, these data also suggest that the recognition of dynamic facial expressions is enhanced compared to that of static expressions in individuals with PDD. Thus, findings on the recognition of static and dynamic facial expressions in PDD are inconsistent. In order to clarify facial emotion processing impairments specific to PDD, it is necessary to examine each component of the dynamic facial expression recognition process.

Little is known, however, about earlier perceptual processing for dynamic facial expressions in individuals with PDD. A recent study of typically developing participants (Yoshikawa and Sato 2008) identified the representational momentum (RM) for dynamic facial expression. RM describes a phenomenon in which the final position of a moving object shifts in the direction of the observed movement in the perceiver's mind (Freyd and Finke 1984; Hubbard 1990). This effect has also been reported in the transformation of object shape (e.g., Kelly and Freyd 1987). Thus, Yoshikawa and Sato found that participants perceived the last image presented during the dynamic facial expression in an exaggerated form. They suggested that the RM for dynamic facial expressions is an adaptive mechanism for detecting subtle changes in another's facial expression. Identifying the reasons individuals with PDD find it difficult to communicate with others via emotional facial expressions would require clarification of whether this mechanism that perceives dynamic facial expressions to be exaggerated is impaired in these individuals.

In this study, we investigated RM for dynamic facial expressions among individuals with highfunctioning PDD and age-matched typically developing controls, using a paradigm set out in a previous study (Yoshikawa and Sato 2008). Briefly, we presented dynamic and static images of facial expressions and asked participants to match the changeable emotional face display with the perceived last image of dynamic facial expression and static facial expression stimuli. Based on the evidence described above (Gepner et al. 2001; Tardif et al. 2007), we hypothesized that both 
individuals with PDD and typically developing controls would perceive the last image in the dynamic facial expression to be more exaggerated than the last static facial expression.

\section{Methods}

\section{Participants}

Participants included 13 individuals with PDD and 13 typically developing controls. The two groups (PDD and control) were matched for chronological age (PDD group: $M=19.0$ years, $S D=$ 5.7; control: $M=19.8$ years, $S D=2.7$; independent t-test, $t(24)=0.44, p>0.1)$ and gender (PDD group: 12 males, 1 female; control: 12 males, 1 female). Verbal and performance IQ in the PDD group was measured using the Japanese version of the WAIS-R (Shinagawa et al. 1990), WAIS-III (Fujita et al. 2006), WISC-R (Kodama et al. 1982), or WISC-III (Azuma et al. 1998). All participants withPDDhad IQs within the normal range (Fullscale IQ: $M=101.8, S D=12.9$; Verbal IQ: $M=104.4, S D=15.5$; Performance IQ: $M=98.3, S D=10.6$ ).

Participants in the PDD group were diagnosed with either Asperger's disorder (4 males, 1 female) or a pervasive developmental disorder not otherwise specified (PDDNOS; 8 males) by a child psychiatrist using DSM-IV-TR (APA 2000). All were outpatients who had been referred to Kyoto University Hospital or the Faculty of Human Health Science of Kyoto University Graduate School of Medicine because of their social maladaptation. They were all free of neurological or psychiatric problems other than those associated with PDD, and none was taking any medication.

The diagnosis of PDD was based on (1) an interview; (2) information from each subject; and (3) childhood clinical records. More specifically, the diagnostic procedure in this study was as follows. First, research assistants (clinical psychologists) collected information from parents on developmental milestones (including joint attention behaviors) and episodes (e.g., how the individual with PDD behaved at school). Information about detailed observations of interactions 
with people (particularly non-family members) as well as repetitive behaviors, obsessive/ compulsive traits, and stereotyped behaviors, was also provided by teachers or other professionals (such as occupational counselors and mental health counselors). An expert child psychiatrist (one of the authors, MT) interviewed each participant in the PDD group at least three times (each on a separate day, with a between-interview interval of more than 2 weeks) before the final diagnosis was made.

All participants aged 18 years and older and the parents of participants aged younger than 18 years provided written informed consent to participate in this study in accordance with the Declaration of Helsinki.

\section{Design}

The experiment was constructed as a three-factorial mixed randomized-repeated design, with group (PDD or control) as the randomized factor, and presentation condition (dynamic or static) and emotion condition (fearful or happy) as the repeated factor.

\section{Stimuli}

From a set of facial images (Ekman and Friesen 1976), we selected one neutral expression slide and two emotional expression (fearful and happy) slides for each of four actors (two men and two women). We used computer morphing techniques (Mukaida et al. 2000) to produce images that were intermediate between the neutral expression and each of the two emotional expressions in $4 \%$ steps. We produced dynamic facial expression stimuli that changed from $4 \%$ emotional expression to a maximum of $80 \%$ of the full emotional expression. We presented a total of 20 image frames in succession, i.e., the first image ( $4 \%$ of the full emotional expression image), 18 intermediate images (from 8 to $76 \%$, in $4 \%$ steps), and the final image (80\%). Figure 1 shows the first image, some intermediate images, and the final image of a dynamic stimulus. Stimuli duration was $200 \mathrm{~ms}$ (10 $\mathrm{ms} /$ frame). In the static condition, emotional expressions (at 80\%) were presented for $200 \mathrm{~ms}$. 


\section{Apparatus}

Stimulus presentation and data acquisition were controlled using a program written in Visual C+ + 5.0 (Microsoft) on a Windows computer (HP xw4300 Workstation). Stimuli were presented on a 17-inch CRT monitor (Iiyama; screen resolution 1,024 × 768 pixels; refresh rate $100 \mathrm{~Hz}$ ). The distance between the monitor and participants was fixed at approximately $57 \mathrm{~cm}$ using a headrest.

\section{Procedure}

Figure 2 shows the trial sequence. On the monitor, the left window was used for stimulus presentation and the right window was used for participant responses. Visual angles of the stimulus and response windows were $11.1^{\circ} \times 7.8^{\circ}$, respectively. In each trial, a dynamic or static stimulus was presented in the stimulus window, and $250 \mathrm{~ms}$ later, an initial face image was presented in the response window. Participants were instructed to match the image in the response window exactly with the last image shown in the dynamic or static stimulus by using the mouse to drag a slider to the left or right. This procedure enabled us to obtain participants' perceptual images more precisely than the typical RM paradigm in which participants need to select, from a limited number of prepared images, one that they think best matches their perceptual image. The face shown in the initial image in the response window had an emotional expression with 70,80 , or $90 \%$ intensity. Slider scales had one of three predefined ranges, each of which had a $100 \%$ range of emotional intensity (i.e., 20-120, 30-130, or 40-140\%). These slider scale ranges varied randomly across trials, and slider ranges were not visible to participants. After a participant selected an image, he or she clicked the button, and the image in the response window disappeared. Then, the stimulus was presented again in the left window, and $250 \mathrm{~ms}$ later, the image chosen by the participant appeared in the response window. If the participant thought the images matched, he or she clicked the button on the display and went on to the next trial; if not, the participant could modify the image until it matched. This second exposure was introduced to avoid a potential problem with participants' responding too slowly upon the first exposure to maintain their perceptual images, which may 


\section{J Autism Dev Disord}

decay over time. A total of 32 trials ( 8 trials per condition) were performed in blocks, and the order of trials was counterbalanced across participants. Before starting the experiment, each participant was given several practice trials and allowed to practice image manipulation using the mouse to move the slider.

\section{Data analysis}

Data were analyzed using SPSS 10.0J (SPSS Japan). For each participant, the average intensity of selected images was calculated for each condition and analyzed with a 2 (group) $\times 2$ (presentation) $\times 2$ (emotion) repeated measures analysis of variance (ANOVA). If any significant interaction was observed, a follow-up simple effect analysis was conducted.

\section{Results}

The ANOVA revealed a significant main effect of presentation condition $(F(1,24)=28.82, p<$ 0.01), indicating that the selected images had a higher emotional intensity in the dynamic facial expression condition than in the static condition. The confirmation analysis verified that both PDD and control groups perceived the last dynamic facial expression image to be more exaggerated than the static facial expression (PDD group: $F(1,24)=19.88, p<0.01$; control group: $F(1,24)=$ $9.97, p<0.01)$. No other significant main effect or interactions were observed (emotion: $F(1,24)=$ $0.03, p=0.87$; emotion $\times$ presentation: $F(1,24)=2.56, p=0.12$; group $\times$ emotion: $F(1,24)=0.11$, $\mathrm{p}=0.74$; group $\times$ presentation: $F(1,24)=0.88, p=0.36$; group $\times$ emotion $\times$ presentation: $F(1,24)$ $=0.51, p=0.48)$. 


\section{Discussion}

The results showed that typically developing controls perceived the last image of dynamic facial expression to be more exaggerated than a static stimulus. This indicates that dynamic presentation of a facial expression elicits RM. These results for the control group support previous findings (Yoshikawa and Sato 2008).

More importantly, the results show that individuals with PDD also perceived dynamic facial expressions to be exaggerated; this is the first study to show that dynamic presentation of a facial expression induces RM among individuals with PDD, just as it does among typically developing controls. The results are consistent with those of previous studies investigating the processing of dynamic facial expressions in PDD populations. Gepner et al. (2001) have found relatively good recognition of facial expressions in dynamic presentation conditions among individuals with PDD, although overall recognition of facial expressions in individuals with PDD is worse relative to controls. Also, Tardif et al. (2007) have shown that slow, dynamic presentation of facial expressions benefits facial expression recognition in individuals with PDD, although these participants were less able than controls to recognize dynamic and static facial expressions. These findings seem to support the results of the present study, indicating that dynamic presentation enhances the processing of facial expressions. Furthermore, the present study showed that individuals with PDD appeared to have a subjective perception of the intensity of dynamic facial expression that was comparable to that of typically developing controls, and that the extent to which dynamic presentation enhanced subjective perception was indistinguishable between individuals with and those without PDD. Taken together, the results appear to indicate that individuals with PDD have an intact ability to process dynamic information from facial cues, at least on a perceptual level.

Our results suggest that individuals with PDD have an intact ability to perceive dynamic facial expressions of emotion. A question arises therefore: what else might be the impaired component in 
facial expression processing in individuals with PDD? A previous study (Corona et al. 1998) has provided clues regarding this issue. In that study, the researchers found that when an experimenter showed either distressed or neutral facial expressions dynamically with vocal sounds, individuals with PDD looked at distressed more than at neutral expressions. These data indicated that they detected dynamic emotional facial expressions, which corroborates our result. However, the individuals with PDD did not show higher autonomic and behavioral responses to distressed than to neutral expressions, although such responses were shown by control participants. These data suggest that individuals with PDD are impaired in their emotional reactions to dynamic facial expressions. Some researchers have proposed that emotional reactions in response to facial expressions are useful for accurately recognizing facial expressions (e.g., Adolphs 2002). Previous studies have suggested differences in performance between individuals with PDD and normal controls in the recognition of dynamic facial expression (Gepner et al. 2001; Tardif et al. 2007). Some studies have indicated that although individuals with PDD have a limited ability to recognize emotional states from a point-light motion display, they are able to recognize actions from the same source (Moore et al. 1997; Hubert et al. 2007). Based on these data and the present results, we speculate that the processing of dynamic facial expressions may be intact at a perceptual level, but impaired at the stage in which emotional reactions to dynamic facial expressions are formed. Such impairment might lead to poor emotion recognition even with dynamic facial expressions. Considering the different methodologies used in the aforementioned studies, however, it is possible that another mechanism may explain the previous and present findings.

The present study has some implications for brain functions in individuals with PDD. Researchers have speculated that the neural mechanism for RM includes an interaction between the dorsal and ventral visual stream including the medial temporal lobe (MT) and the superior temporal sulcus (STS) (Kourtzi and Kanwisher 2000; Senior et al. 2000). In addition, consistent with the present findings, previous neuroimaging studies have revealed that the MT/V5 (Pelphrey et al. 
2007) and STS (Pelphrey et al. 2005) are activated normally in individuals with PDD by dynamic facial expression and gaze shift, respectively. The functioning of these regions might be intact in early perceptual processing of biological motion stimuli such as dynamic facial expressions.

A concern for the present experimental procedure is that matching dynamic expressions with static images might have been more difficult. It has been suggested that the task difficulty can induce large variance in the data (e.g., Elfenbein and Ambady 2002), which may produce pseudo effects. Consistent with this notion, visual inspections of Fig. 3 suggests that response variances in the dynamic conditions were larger than in the static conditions, which may have induced artificial mean differences between conditions. To test this possibility, we first calculated the each participant and then tested the differences. No significant differences were found (although tendencies were shown) for either the PDD or control groups, $t(25)=1.70$ and $1.75, p=0.10$ and 0.09 , respectively. Next, we constructed models in which both mean and variance were different, only variance was different, only mean was different, and neither mean nor variance was different across dynamic and static conditions. The data were assumed to be normally distributed. The comparisons among these models using Akaike information criteria (AIC; Sakamoto et al. 1986) showed that the first model fitted the data better than did the second, third, or fourth model for both PDD and control groups $(\mathrm{AIC}=189.42,195.97,191.56$, and 198.61 for PDD groups and 173.25, 179.73, 173.94, and 180.77 for control groups, respectively). Taken together, these results suggest that dynamic presentations of facial expressions, when compared to static presentations, induced a systematic increase in the matching of final images, irrespective of response variances.

This study had some limitations. An important one may be that the clinical group included only individuals with high-functioning PDD. Gepner (2004) suggested that motion processing is more impaired in individuals with low- than high-functioning PDD. Further research is needed to determine whether the results can be extended to individuals with lower-functioning PDD. 
In summary, this study demonstrated that individuals with PDD, like typically developing controls, clearly exhibit RM for dynamic facial expressions. This finding suggests that individuals with high-functioning PDD have an intact mechanism that perceives dynamic facial expressions to be exaggerated. 


\section{References}

Adolphs, R. (2002). Neural systems for recognizing emotion. Current Opinion in Neurobiology, 12, $169-177$.

Adolphs, R., Sears, L., \& Piven, J. (2001). Abnormal processing of social information from faces in autism. Journal of Cognitive Neuroscience, 13, 232-240.

American Psychiatric Association. (2000). Diagnostic and statistical manual for mental disorders (DSM-IV-TR). Washington,DC: APA.

Azuma, H., Ueno, K., Fujita, K., Maekawa, H., Ishikuma, T., \& Sano, H. (1998). Japanese Wechsler intelligence scale for children-III. Tokyo: Nihon-Bunka-Kagaku-sha.

Bould, E., Morris, N., \& Wink, B. (2008). Recognising subtle emotional expressions: The role of facial movements. Cognition and Emotion, 22, 1569-1587.

Castelli, F. (2005). Understanding emotions from standardized facial expressions in autism and normal development. Autism, 9, 428-449.

Corden, B., Chilvers, R., \& Skuse, D. (2008). Avoidance of emotionally arousing stimuli predicts social-perceptual impairment in Asperger's syndrome. Neuropsychologia, 46, 137-147.

Corona, R., Dissanayake, C., Arbelle, S., Wellington, P., \& Sigman, M. (1998). Is affect aversive to young children with autism? Behavioral and cardiac responses to experimenter distress. Child Development, 69, 1494-1502.

Ekman, P., \& Friesen, W. V. (1976). Pictures of facial affect. Palo Alto, CA: Consulting Psychologists Press.

Elfenbein, H. A., \& Ambady, N. (2002). On the universality and cultural specificity of emotion recognition: A meta-analysis. Psychological Bulletin, 128, 203-235.

Freyd, J. J., \& Finke, R. A. (1984). Representational momentum. Journal of Experimental 
Psychology: Learning, Memory and Cognition, 10, 126-132.

Fujita, K., Maekawa, H., Dairoku, H., \& Yamanaka, K. (2006). Japanese Wechsler adult intelligence scale-III. Tokyo: Nihon-Bunka-Kagaku-sha.

Gepner, B. (2004). Autism, movement, and facial processing. The American Journal of Psychiatry, $161,1719$.

Gepner, B., Deruelle, C., \& Grynfeltt, S. (2001). Motion and emotion: A novel approach to the study of face processing by young autistic children. Journal of Autism and Developmental Disorders, 31, 37-45.

Hubbard, T. L. (1990). Cognitive representation of linear motion: Possible direction and gravity effects in judged displacement. Memory and Cognition, 18, 299-309.

Hubert, B., Wicker, B., Moore, D. G., Monfardini, E., Duverger, H., Da Fonseca, D., et al. (2007). Recognition of emotional and nonemotional biological motion in individual with Autistic Spectrum Disorders. Journal of Autism and Developmental Disorders, 37, 1386-1392.

Kelly, M. H., \& Freyd, J. J. (1987). Explorations of representational momentum. Cognitive Psychology, 19, 369-401.

Kodama, H., Shinagawa, F., \& Motegi, M. (1982). Japanese Wechsler intelligence scale for children—revised. Tokyo: Nihon-Bunka-Kagaku-sha.

Kourtzi, Z., \& Kanwisher, N. (2000). Activation in human MT/MST by static images with implied motion. Journal of Cognitive Neuroscience, 12, 48-55.

Moore, D. G., Hobson, R. P., \& Lee, A. (1997). Components of person perception: An investigation with autistic, non-autistic retarded, and typically developing children and adolescents. British Journal of Developmental Psychology, 15, 401-423.

Mukaida, S., Kamachi, M., Kato, T., Oda, M., Yoshikawa, S., \& Akamatsu, S. (2000). Foolproof utilities for facial image manipulation [unpublished computer software]. Kyoto: ATR.

Pelphrey, K. A., Morris, J. P., \& McCarthy, G. (2005). Neural basis of eye gaze processing deficits 
in autism. Brain, 128, 1038-1048.

Pelphrey, K. A., Morris, J. P., McCarthy, G., \& Labar, K. S. (2007). Perception of dynamic changes in facial affect and identity in autism. Social Cognitive and Affective Neuroscience, 2, 140-149.

Sakamoto, Y., Ishiguro, M., \& Kitagawa, G. (1986). Akaike information criterion statistics. Tokyo: KTK Scientific Publishers.

Sato, W., \& Yoshikawa, S. (2007). Enhanced experience of emotional arousal in response to dynamic facial expressions. Journal of Nonverbal Behavior, 31, 119-135.

Senior, C., Barnes, J., Giampietro, V., Simmons, A., Bullmore, E. T., Brammer, M., et al. (2000). The functional neuroanatomy of implicit-motion perception or representational momentum. Current Biology, 10, 16-22.

Shinagawa, F., Kobayashi, D., Fujita, K., \& Maekawa, H. (1990). Japanese Wechsler adult intelligence scale-revised. Tokyo: Nihon-Bunka-Kagaku-sha.

Tardif, C., Laine才, F., Rodriguez, M., \& Gepner, B. (2007). Slowing down presentation of facial movements and vocal sounds enhances facial expression recognition and induces facial-vocal imitation in children with autism. Journal of Autism and Developmental Disorders, 37, 1469_ 1484.

Yoshikawa, S., \& Sato, W. (2008). Dynamic facial expressions of emotion induce representational momentum. Cognitive, Affective, and Behavioral Neuroscience, 8, 25-31. 
Author Note

Shota Uono: Department of Cognitive Psychology in Education, Faculty of Education, Kyoto University, Yoshida-honmachi, Sakyo-ku, Kyoto 606-8501, Japan. Wataru Sato: Department of Comparative Study of Cognitive Development (Funded by Benesse Corporation), Primate Research Institute, Kyoto University, Inuyama, Aichi 484-8506, Japan. Motomi Toichi: Faculty of Human Health Science, Graduate School of Medicine, Kyoto University, Shogoin Kawahara-cho, Sakyo-ku, Kyoto 606-8507, Japan.

Correspondence concerning this article should be addressed to Shota Uono (Department of Cognitive Psychology in Education, Faculty of Education, Kyoto University, Sakyo-ku, Kyoto, 606-8501, Japan. Tel: +81-75-753-3067; Fax: +81-75-753-3067; E-mail: shotauono@p06.mbox.media.kyoto-u.ac.jp). 
Figure Captions

Figure 1. Examples of the morphing image sequence for dynamic facial expression of emotion.

Figure 2. The trial sequence.

Figure 3. The mean percentage of selected images in each condition. 


\section{Figure 1.}

\section{Dynamic}

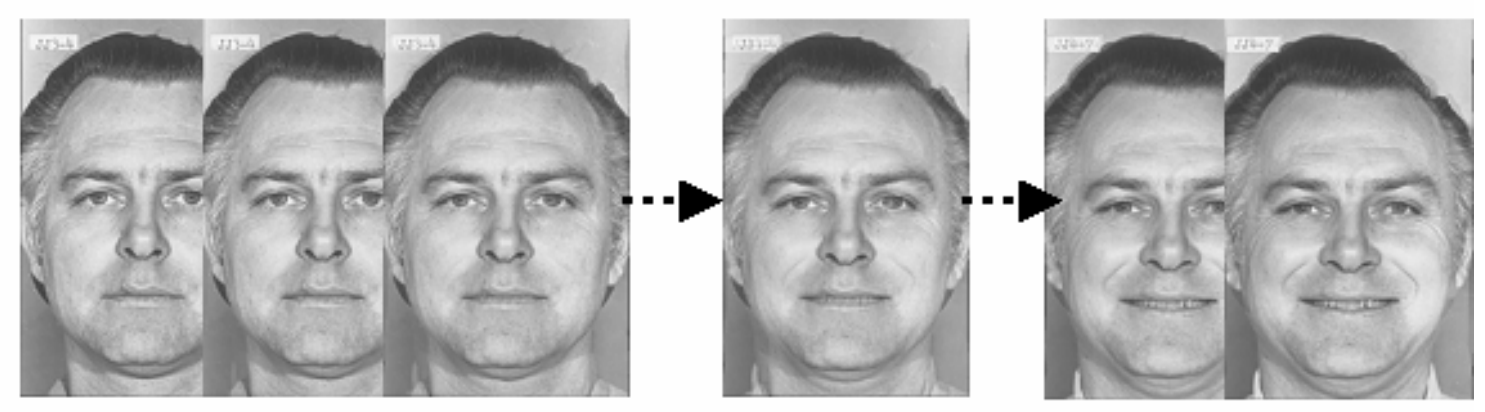

\section{Static}

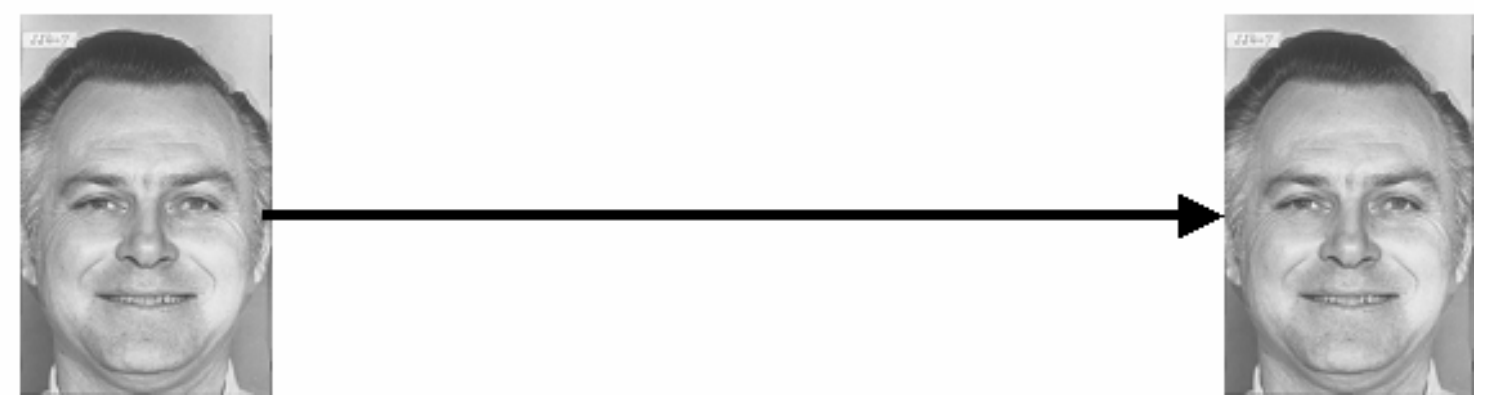


J Autism Dev Disord

\section{Figure 2.}
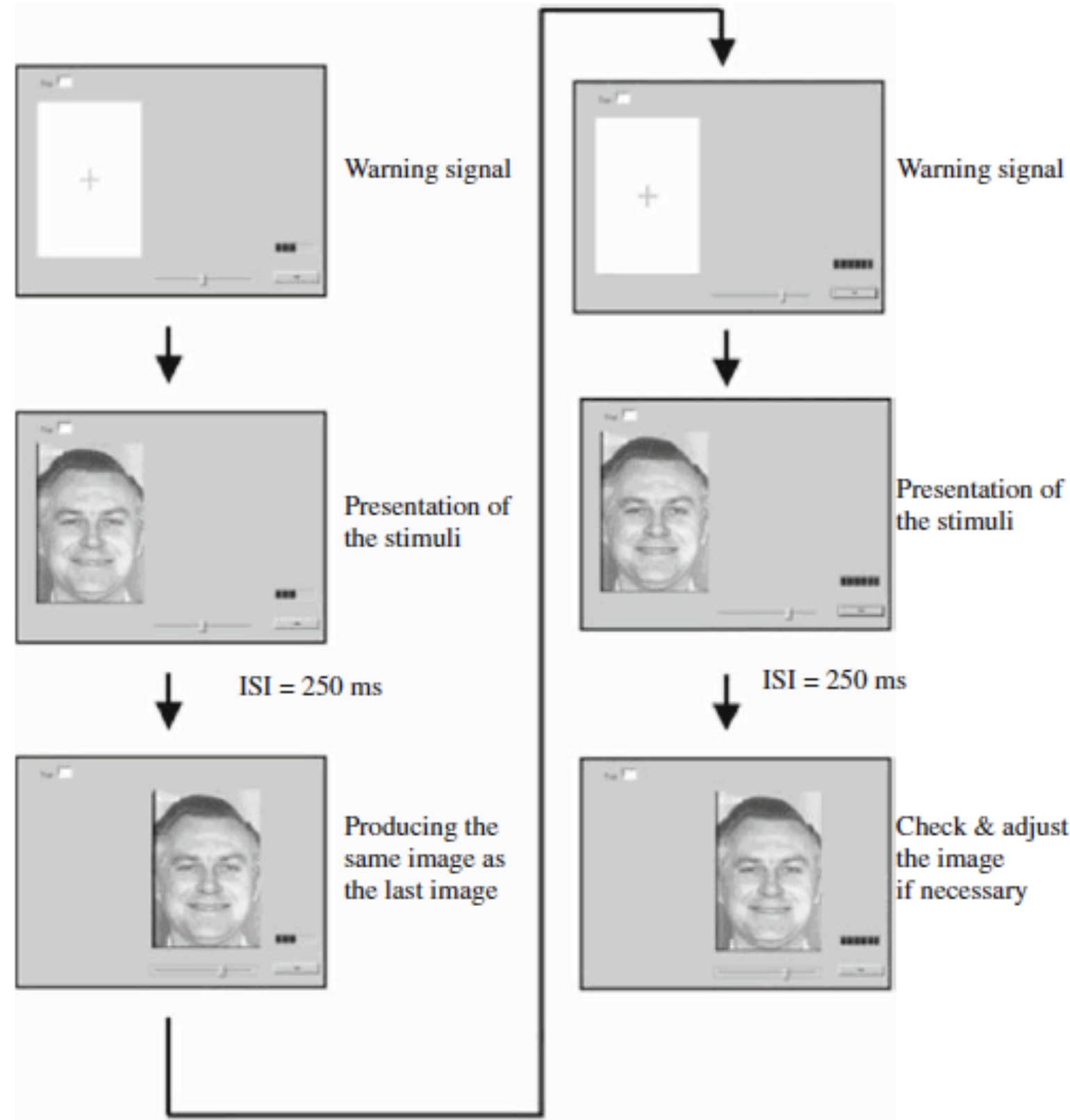

$\Rightarrow \quad$ Key press 
Figure 3.

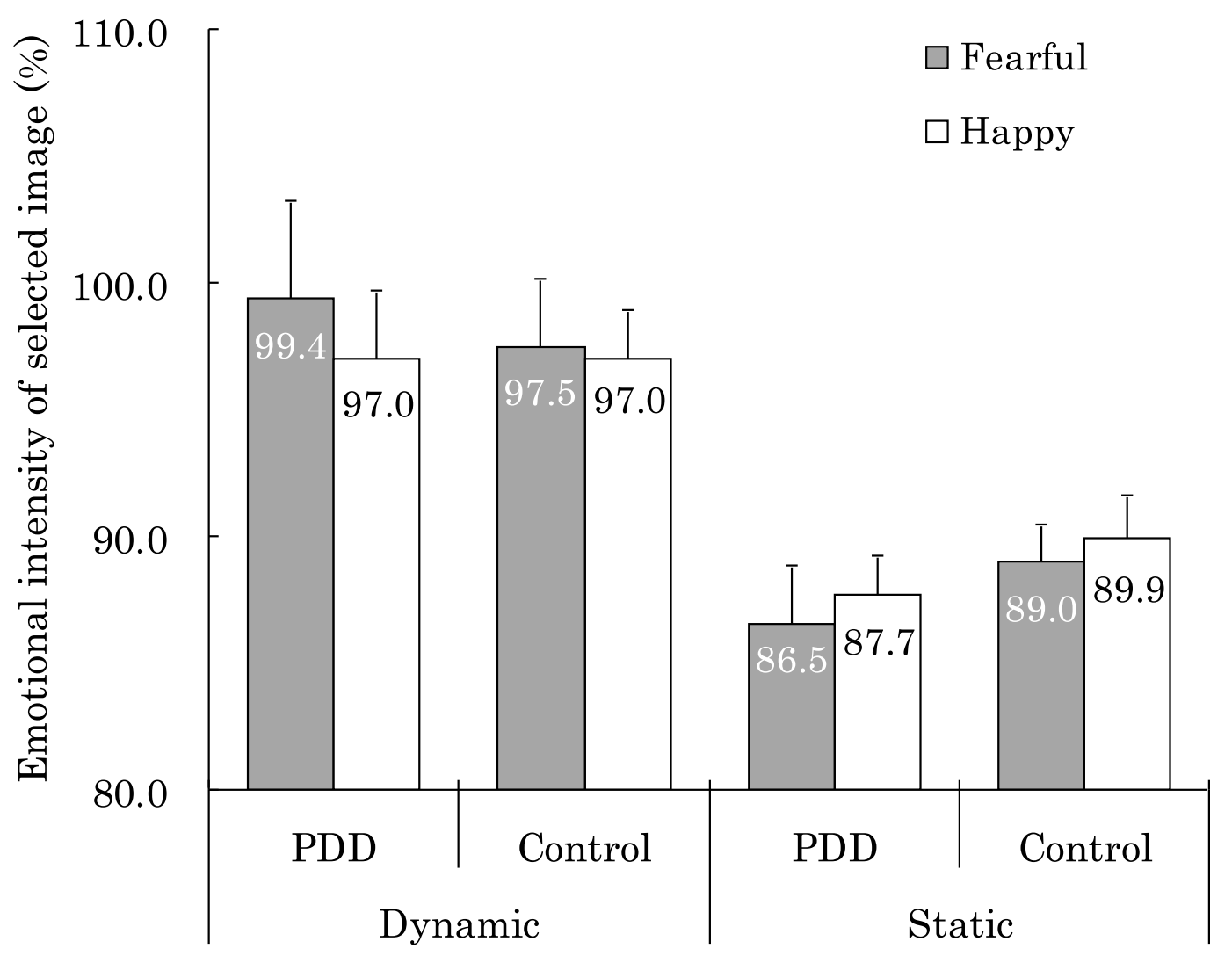

